\title{
Gamificación en educación superior. Una scape room para el aula de matemáticas
}

\section{María García Monera}

Departamento de Matemáticas, área de Geometría y Topología, Universitat de València, C/ Doctor Moliner 50, 46100, Burjassot, monera2@uv.es

\begin{abstract}
For some years now, the profile of our students has changed considerably. This means that, in a complementary way to the master classes, teachers gradually have to adapt their lessons to new generations, much more technological than years ago. One of the ways to increase the motivation and participation of the students in our subject is to plan and carry out different activities so that this also help us to develop materials that are suitable for as many students as possible. The general objective of this article is to use gamification as a teaching tool in the classroom. Specifically, we propose the use of the scape room in the subject of mathematics in higher education. Increasingly, these types of resources are being used to help students develop social skills, encourage teamwork, and streamline classes beyond master classes. 23 students participated in the study as a pilot group from the faculty of Biology of the University of Valencia. After the completion of the activity, it has been decided to implement the activity in the following academic years and extend it to all the students enrolled in the subject Mathematics I. It is also intended in future sessions to extend the experience to other departments that teach in the same degree, in order to create a scape room based in different contexts.
\end{abstract}

Keywords: gamification, learning, mathematics, scape room

\section{Resumen}

De unos años a esta parte, el perfil de nuestros estudiantes ha cambiado de manera considerable. Esto hace que, de manera complementaria a las clases magistrales, los profesores poco a poco debamos adaptarnos a las nuevas generaciones, mucho más tecnológicas que años atrás. Una de las maneras de aumentar la motivación y la participación de los estudiantes en nuestra asignatura es el planteamiento y la realización de diferentes actividades de manera que esto nos ayude también a desarrollar materiales que se adecuen al mayor número de alumnos posible. El objetivo general del presente artículo es utilizar la gamificación como herramienta docente en el aula. En concreto, proponemos el uso de la scape room en el aula de matemáticas en la enseñanza superior. Este tipo de recursos se están utilizando cada vez más a fin de ayudar a los estudiantes a desarrollar habilidades sociales, fomentar el trabajo en equipo y dinamizar las clases más allá de las clases magistrales. En el estudio participaron 23 estudiantes como grupo piloto de la facultad de Biología de la Universitat de València. Después de la realización de la actividad se ha decido implementar la actividad en los siguientes cursos académicos y extenderlo a todo el alumnado matriculado en la asignatura Matemáticas I. También se pretende en próximas sesiones extender la experiencia a otros departamentos que impartan docencia en el grado, a fin de poder realizar una scape room ambientada en diferentes contextos.

Palabras clave: aprendizaje, gamificación, habitación de escape, matemáticas. 


\section{Introducción}

El desarrollo tecnológico es una realidad a la que poco a poco los profesores universitarios nos hemos tenido que ir acostumbrando. Ya no solo por el hecho de implementar en nuestras clases el uso de software específico de nuestras asignaturas, si no por cómo este desarrollo tecnológico está afectando a las nuevas generaciones de estudiantes que se matriculan en los primeros cursos de grado de nuestra universidad. De unos años a esta parte, ya en las primeras etapas de la educación secundaria y en bachiller, incluso en infantil y primaria, se está fomentado el uso de las TIC como recursos educativos. Comienza a ser común en numerosos centros educativos encontrar pizarras digitales en las aulas, impresoras 3D, incluso tabletas y teléfonos móvil. Esto hace que el perfil de alumnado que llega a la universidad haya cambiado considerablemente al que nos podríamos encontrar años atrás. Hoy en día, los estudiantes están más que acostumbrados a hacer uso de los recursos tecnológicos que tienen a su alcance. Las visitas a las bibliotecas poco a poco se van sustituyendo por video tutoriales on-line que los estudiantes visualizan antes de comenzar a resolver una tarea (Unicoos). Esto hace que, en conjunto con las clases magistrales en la universidad, los profesores debamos ir adaptándonos poco a poco a las nuevas generaciones.

Desde hace algunos años están empezando a surgir nuevas metodologías de enseñanza adaptando las nuevas tecnologías, pero generalmente basadas en metodologías anteriores. Un ejemplo de ello es la que se conoce como gamificación. El término gamificación proviene de la palabra inglesa "game" y hace referencia al uso de elementos propios del juego, pero en un entorno profesional o educativo a fin de obtener mejores resultados. El objetivo principal es atraer al jugador e incitarle a la acción y promover el aprendizaje resolviendo problemas con dinámicas propias del juego (Diago y Ventura, 2017). Este tipo de técnicas, cada vez más comunes en el ámbito educativo, han dado lugar a lo que se conoce como gamificación educativa. Esta metodología permite al estudiante tener un papel más activo en el proceso de enseñanza-aprendizaje y evita que sea un simple receptor de conocimiento. Además, ayuda al estudiante a buscar su propia autonomía, algo que persigue el Espacio Europeo de Educación Superior (EEES). Numerosos estudios demuestran que la gamificación ayuda a desarrollar nuevas competencias y a mejorar la motivación y cohesión entre los estudiantes (Zhang et al. 2018).

Un ejemplo de gamificación educativa lo encontramos en las scape room o habitación de escape. Las scape room son una dinámica de aventura real en la que los participantes han sido encerrados en una sala de la cual deben conseguir escapar. A través de la resolución de problemas, acertijos o enigmas a modo de pruebas, los estudiantes deben llegar al final de juego, es decir, conseguir encontrar la forma de salir. La idea original surgió en Kyoto, Japón (Diago y Ventura, 2017) y en la actualidad miles de empresas en todo el mundo ofrecen diferentes tipos de scape rooms de diferentes temáticas. Un ejemplo del éxito adquirido por este tipo de actividades es que sólo en la ciudad de Valencia ya encontramos cerca de 20 empresas dedicadas a las habitaciones de escape.

En el presente trabajo exponemos el desarrollo de una scape room llevada a cabo en el aula de matemáticas a nivel universitario. Antes de llevar a cabo una scape room, al igual que cualquier otra actividad que queramos implementar en el aula, es importante tener una planificación previa, que en nuestro caso desarrollaremos a lo largo del artículo. Esta planificación debe incluir:

- Objetivos: los objetivos son las intenciones que guían el proceso de enseñanza-aprendizaje y que los alumnos deben adquirir al final del proceso.

- Contenido curricular: el contenido curricular hace referencia a la parte del temario se pretende reforzar o ampliar con la actividad que se va a llevar a cabo en el aula. 
- Temporalización: Es muy importante tener una buena planificación antes de la realización de la actividad. No sólo es importante saber cómo vamos a distribuir el tiempo ese día a lo largo de la experiencia, sino también saber cuándo se va a llevar a cabo. Los contenidos que queramos trabajar con los estudiantes serán los que nos marcarán el cuándo y de qué manera vamos a realizar la actividad.

- Materiales y espacios: Una vez decididas las actividades que van a realizar los estudiantes, debemos tener claro el tipo de materiales que vamos a necesitar y, sobre todo, dónde se va a realizar.

- Argumento: Todas las scape room o habitaciones de escape requieren de un hilo conductor en el que están ambientadas las actividades. Antes de comenzar la actividad deberá explicarse a los estudiantes en qué consiste y cuál es el objetivo final.

El presente artículo está estructurado de la siguiente forma: en la primera sección presentamos los objetivos de la experiencia docente que nos planteamos antes de llevarla a cabo. En el siguiente apartado desarrollamos la innovación explicando cómo se realizó y el tipo de problemas que se les plantearon a los estudiantes. Finalmente, la última parte está dedicada a los resultados y conclusiones.

\section{Objetivos}

El objetivo principal de esta experiencia educativa fue evaluar la eficacia de una scape room en el proceso de aprendizaje de los estudiantes. Partiendo de la premisa de que se realizó la actividad con alumnos de la facultad de Biología de la Universitat de València, esto hacía que, a priori, los estudiantes no tuvieran gran interés por las matemáticas. Es por ello que nos planteamos los siguientes objetivos:

- Potenciar el interés de los estudiantes por las matemáticas.

- Elaborar material docente que se pueda incorporar en los siguientes cursos académicos.

- Dar a conocer a los estudiantes otras metodologías docentes.

- Fomentar el trabajo en equipo de los estudiantes ayudando a desarrollar sus habilidades sociales y liderazgo.

- Contribuir a la formación científica de los estudiantes.

\section{Desarrollo de la innovación}

La propuesta de este trabajo se ha desarrollado en el presente curso 2019/2020 dentro de la asignatura Matemáticas I del grado en Bioquímica y Ciencias Biomédicas de la Universitat de València. Esta asignatura obligatoria tiene una carga lectiva de 6 créditos ECTS repartidos entre las sesiones de teoría, prácticas y tutorías presenciales. Los contenidos que se trabajan en la asignatura son:

- Tema 1: Geometría básica del plano y el espacio

- Tema 2: Funciones y límites

- Tema 3: La derivada

- Tema 4: Aplicaciones de las derivadas. Máximo y mínimos

- Tema 5: La integral de funciones de una variable

- Tema 6: La integral definida de funciones de una variable

- Tema 7: Ecuaciones diferenciales ordinarias

- Tema8: Algunas ecuaciones diferenciales ordinarias en Biología y Medio Ambiente

(c) 2020, Universitat Politècnica de València

Congreso In-Red (2020) 
De los 82 alumnos matriculados, se tomó una muestra de 23 alumnos, correspondientes a uno de los grupos de prácticas.

\subsection{Contenido curricular}

Antes de la realización de cualquier actividad, es importante saber qué parte del temario se pretende reforzar o ampliar. En nuestro caso, la intención era reforzar los primeros temas del curso, por lo que desarrollamos la actividad después del tema 4. En ella trabajamos:

- Representación de funciones.

- Máximos y mínimos y discontinuidades de una función.

- Máximos y mínimos en el contexto de la Biología.

Esto hizo que la actividad se llevara a cabo una vez finalizado el tema 4, es decir, a finales de noviembre del presente curso escolar.

\subsection{Temporalización}

La temporalización es otro punto importante antes de la realización de la actividad. Es importante tener clara la distribución del tiempo que se va a llevar: Explicación de la actividad, desarrollo y conclusiones o feedback por parte del alumnado.

Nuestra actividad se llevó a cabo durante una de las sesiones de prácticas, con una duración de $2 \mathrm{~h}$. Además, dado que los alumnos necesitaban hacer uso de software Mathematica, un potente programa informático para la realización de problemas, esto requirió de unas sesiones previas para el aprendizaje del manejo del programa. Con respecto a la distribución horaria dentro de la actividad, ésta fue la siguiente forma:

- 10 minutos de preliminares donde explicamos en qué consistía la scape room,

- $\quad 1 \mathrm{~h} 40 \mathrm{~min}$ a la realización de las actividades.

- 10 minutos para conclusiones o feedback por parte de los estudiantes.

\subsection{Materiales y espacios}

En nuestro caso, fue necesario adquirir previamente varias cajas de madera de distintos tamaños, así como candados y algunas golosinas a modo de premio. La actividad se llevó a cabo en un aula de informática, ya que los alumnos necesitaban hacer uso del ordenador. Además, los alumnos también necesitaron de su propio móvil, ya que en una de las actividades necesitaban escanear un código QR.

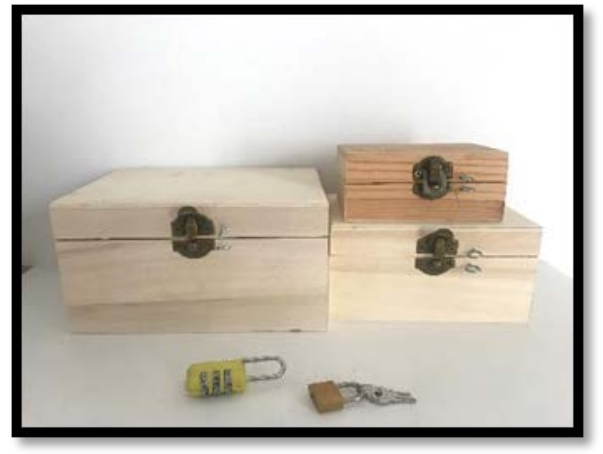

Fig. 1 Material utilizado por cada uno de los grupos 


\subsection{Argumento de la scape room}

Tal y como comentábamos anteriormente, todas las habitaciones de escape están ambientadas en alguna situación particular. El hilo conductor de nuestra actividad fue la "salvación" de la rectora de la Universitat de València. La rectora había sido secuestrada y varias bombas se había distribuido por la universidad. Los alumnos, a través de la resolución de problemas, debían encontrar los códigos de desactivación de las bombas y así, finalmente, salvar a la rectora...

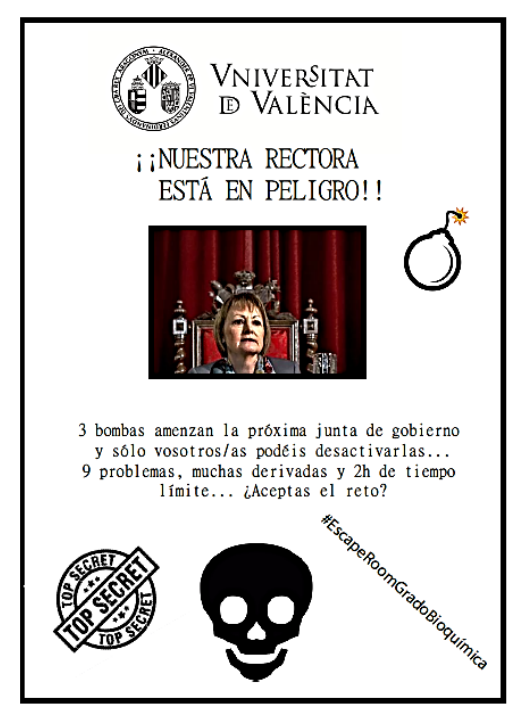

Fig. 1 Imagen del comienzo de la actividad

\subsection{Desarrollo de la scape room}

El día de la realización de la actividad se distribuyó a los alumnos en grupos de 3-4 personas y se les asignó uno de los ordenadores del aula. Una vez tuvimos a los alumnos organizados, proyectamos en el cañón del aula el comienzo de la actividad (Fig. 1) y se les explicó en qué iba a consistir. Cuando tuvieron claro cuál era el fin de la experiencia se les hizo entrega de una caja cerrada con un candado y un folio con la primera actividad a cada uno de los grupos. La primera actividad consistía en la resolución de 3 problemas, donde la solución de cada uno de ellos correspondía a una cifra que nos daría el código del candado. Era importante que cada grupo tuviera su propia contraseña, a fin de que resolvieran por separado los problemas planteados. Por ello, en cada una de las pruebas, cada grupo tenía los mismos problemas, pero con datos diferentes. Esto hacía que la solución fuera diferente y, por tanto, no hubiera opción de que se dijeran la contraseña.

Una vez conseguido el código, los alumnos encontraron dentro de la caja otra más pequeña también cerrada con un candado. En este caso, la caja más pequeña llevaba adjunto un código QR que debían escanear (Fig. 2). 


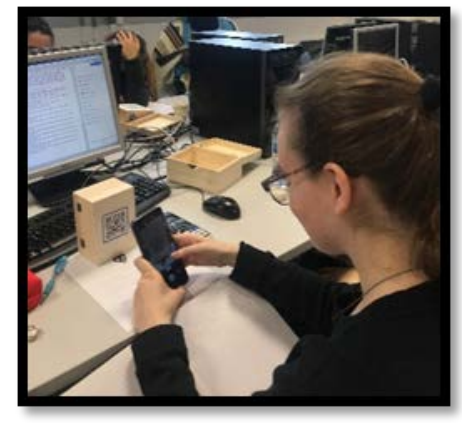

Fig. 2 Alumna escaneando un código $Q R$

Este código QR les llevaba nuevamente a un .pdf con 3 problemas, donde la primera cifra de cada una de las soluciones era de nuevo un dígito de la contraseña. Finalmente, una vez consiguieron abrir la caja, encontraron dentro otra caja más pequeña, esta vez cerrada con un candado con llave y una hoja de instrucciones. Las instrucciones decían que debían hacer una representación de una función matemática cumpliendo una serie de requisitos. Una vez la tuvieran dibujada debían mostrársela al profesor del aula junto con la solución de los problemas anteriores. Esto se hizo a fin de asegurarnos que los estudiantes habían resuelto todos los problemas. Al ser un candado de 3 dígitos, era muy fácil encontrar los 2 primeros y buscar el tercero al azar. Después de asegurarnos que estaba todo correcto, el profesor les hizo entrega de un sobre que contenía una imagen a modo de pista. Por ejemplo, una de las pistas que se entregó a uno de los grupos era la imagen de dos soles (Fig. 3)
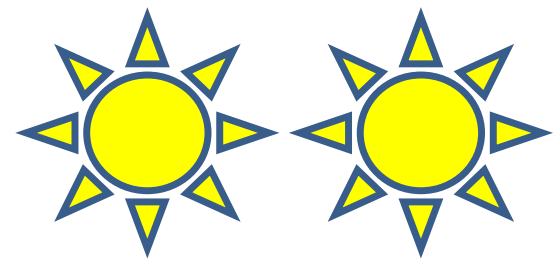

Fig. 3 Última pista de uno de los grupos

En este caso, la llave que abría la última de las cajas se encontraba dentro de un sobre pegado debajo de la mesa donde habían estado trabajando a lo largo de la actividad (caliente, caliente...). Esto se había hecho antes de comenzar la sesión. Para ello, debimos asegurarnos previamente de que el grupo asociado a esta pista se situaba en el lugar correcto. Al conseguir la llave y abrir la caja, los estudiantes encontraron una nota donde se les indicaba que las bombas habían sido desactivadas y algunas golosinas a modo de recompensa.

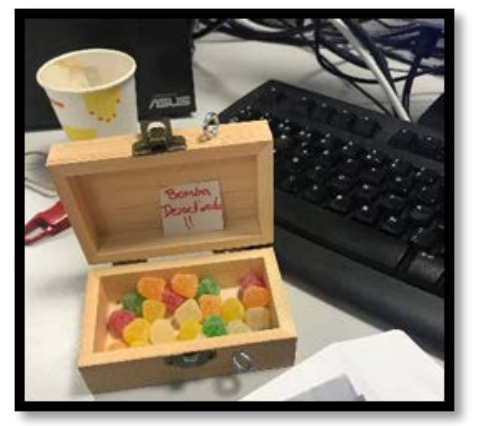

Fig. 4 Final de la prueba 


\section{Resultados}

Conforme los estudiantes iban acabando la actividad se les preguntó por su opinión respecto a la misma. En general, el feedback por parte de los alumnos fue muy enriquecedor, ya que coincidían en calificar la experiencia com divertida, formativa y constructiva. Además, también agradecieron el hecho de la realización de este tipo de actividades grupales en la asignatura de matemáticas, más allá de la clase magistral tan común en esta asigantura.

Por parte del profesorado, destacar que el número de alumnado fue el adecuado y permitió desarrollar la actividad sin complicaciones. El tiempo fue suficiente para que los estudiantes pudieran acabar todas las pruebas sin complicación. Además, al ser una actividad para afianzar conceptos, el profesorado sirvió de guía pero en pocas ocasiones tuvo que ayudar en la resolución de los problemas, ya que era un temario que se había trabajado previamente tanto en clase de teoría como en prácticas.

Si bien es cierto que el número de estudiantes que participaron corresponde únicamente al $30 \%$ del alumando matriculado aproximadamente, en el próximo curso está previsto la ampliación a los diferentes grupos de prácticas, lo que nos permitirá obtener una cantidad de datos fiables a través de una rúbrica con respecto, por un lado, al grado de adquisición de conocimientos por parte de los estudiantes y por otro lado, obtener datos respecto al grado de adquisición de las competencias transversales a través de este tipo de actividades, como puedan ser: trabajo en equipo, liderazgo, habilidades interpersonales o capacidad de aprender (Tuning).

\section{Conclusiones}

Tal y como comentábamos anteriormente, aunque la actividad se llevó a cabo con un grupo reducido de estudiantes, la conclusión general es que la gamificación tiene una gran aceptación entre el estudiantado y aumenta su grado de participación y de motivación.

A lo largo de esta experiencia docente se trabajaron con conceptos y problemas que los alumnos ya habían visto previamente, lo que facilitó su trabajo a lo largo de la misma. En una línea futura de actuación, por un lado, se pretende extender la actividad a todos los alumnos del grupo, realizando la actividad en grupos reducidos y días diferentes, así como realizar la experiencia en colaboración con otros departamentos que impartan docencia al grupo. Esto ayudaría a que la actividad fuera más transversal y enfocada a la enseñanda STEM (Science, Technology, Engineering, Mathematics), tan demandada en estos últimos años. Por otro lado, pretendemos trabajar con conceptos y problemas nuevos que los estudiantes no hayan visto a lo largo de las clases teóricas, lo que aumentaría el grado de complejidad y les permitiría ampliar conocimientos de la materia. Esto también nos permitirá analizar la efectividad de este tipo de actividades grupales a la hora de adquirir nuevos conocimientos.

Finalmente, destacar que las conclusiones derivadas de esta experiencia nos permitirán decidir sobre la inlcusión de la actividad dentro del PIC (Plan de Innovación de Centro) que engloba las actividades de innovación docente que se llevan a cabo a lo largo de los diferentes grados en cada una de las facultades de la Universitat de València. 


\section{Referencias}

\section{Artículo de una revista o periódico}

DIAGO NEBOT, P. y VENTURA CAMPOS, N. (2017). "Escape room: Gamificación educativa para el aprendizaje de las matemáticas" en Suma+, vol. 85, p. 33-40.

ZHANG XC, LEE H, RODRIGUEZ C, RUDNER J, CHAN TM \& PAPANAGNOU D (2018). “Trapped as a Group, scape as a Team: Applying Gamification to Incorporate Team-building Skills Through an 'Escape Room'

Experience”. Cureus. 10, 3, p-e2256. doi: 10.7759/cureus.2256

\section{Referencias electrónicas (libro, revista, o artículo)}

CARREÑO, A., GIMENO SORIANO, M., SANABRIA CODESAL, E. y SIXTOB D. (2019), "Claves para dinamizar una asignatura básica de matemáticas”, In-Red 2019, V Congreso de Innovación Educativa y Docencia en Red, < http://dx.doi.org/10.4995/INRED2019.2019.10487>

GUTIÉRREZ-PRAENA D., RÍOS-REINA R., RUIZ R., TALERO EC., CALLEJÓN R., CALLEJÓN RM., CASAS M., DE LA HABA RR., GARCÍA-MIRANDA P, CARRASCAL L, GUZMÁN-GUILLÉN R (2019), "El uso de una scape room como recurso docente en la Facultad de Farmacia", In-Red 2019, V Congreso de Innovación Educativa y Docencia en Red, < http://dx.doi.org/10.4995/INRED2019.2019.10356>

\section{Página web}

THE UNIVERSITY OF OXFORD. Oxford Mathematics scape room. < https://www.maths.ox.ac.uk/node/32753 > [Consulta: 23 de marzo de 2020]

COEY MATH scape! Using math. < https://coeymath.weebly.com/escape-room.html > [Consulta: 23 de marzo de 2020]

WE ARE TEACHERS So you want to Build a Classrom Scape Room... < https://www.weareteachers.com/build-aclassroom-escape-room-lesson/ > [Consulta: 23 de marzo de 2020]

BREAKOUTEDU. BreakoutEDU. Immersive learning games platform, <http://www.breakoutedu.com/> [Consulta: 23 de marzo de 2020]

TABARA CARBAJO, J. L. (2014) “Curso Mathematica”. YouTube

$<$ https://www.youtube.com/watch? $v=O 2 j h v b q V u G k \& t=231 s>$ [Consulta: 23 de marzo de 2020]

TUNING. 2002. Proyecto Tuning

<https://www.unideusto.org/tuningeu/images/stories/documents/General_Brochure_Spanish_version.pdf. $>$ [Consulta: 23 de marzo de 2020]

UNICOOS “Unicoos”. YouTube https://www.youtube.com/channel/UC3RYy7GbMHDvPQGCdAh3H5g > [Consulta: 23 de marzo de 2020] WOLFRAM, S. Mathematica de wolfram research. <https://www.wolfram.com/mathematica> [Consulta: 23 de marzo de 2020]

\section{Vídeo de Internet}

"Tutorial Escape room educativo en Matemáticas con Realidad aumentada". Youtube < https://www.youtube.com/watch?v=k_dZAkRRcc0 > [Consulta: 23 de marzo de 2020] 